\title{
Correction to: Surgical Management of Symptomatic Lumbar, Sacral, and Lumbosacral Plexus Tumors: a Peripheral Nerve Unit Experience
}

\author{
Fernando Guedes ${ }^{1}$ (D) - Gabriel Elias Sanches ${ }^{1} \cdot$ Rosana Siqueira Brown $^{1} \cdot$ Rodrigo Salvador Vivas Cardoso $^{1}$.

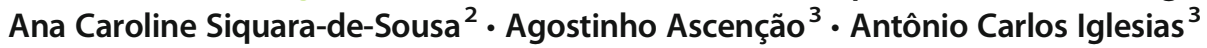

Published online: 29 March 2021

(C) Springer-Verlag GmbH Austria, part of Springer Nature 2021

\section{Correction to: Acta Neurochirurgica https://doi.org/10.1007/s00701-021-04789-0}

The original article is missing the "Comments" below which should be included in the paper.

\section{Comments:}

This paper describes a series of 19 patients with lumbar, sacral and lumbosacral plexus tumors treated at one peripheral nerve unit. These tumors in these locations are relatively rare and the heterogeneity is to be expected; thus, a large experience, successful application of the protocol, and the consequent analysis are not possible. This paper has several strengths which need to be highlighted for general neurosurgeons who have little familiarity with the evaluation and/or management of these lesions.

The authors present an anatomical classification for these tumors and proposes surgical approaches to each of the anatomical regions described. They also offer a useful algorithm that allows standardization for the management and surgery for these patients. We believe that these approaches should be

The online version of the original article can be found at https://doi.org/ 10.1007/s00701-021-04789-0

Fernando Guedes

neuroguedes@yahoo.com.br

1 Department of Surgery, Division of Neurosurgery, Gaffrée e Guinle University Hospital, School of Medicine, Federal University of Rio de Janeiro State (UNIRIO), 775 Mariz e Barros Street, Rio de Janeiro, RJ 20270-901, Brazil

2 Department of Pathology, Antônio Pedro University Hospital, Fluminense Federal University (UFF), Niterói, RJ, Brazil

3 Department of Surgery, Gaffrée e Guinle University Hospital, School of Medicine, Federal University of Rio de Janeiro State (UNIRIO), Rio de Janeiro, RJ, Brazil incorporated in our care of these patients with these regional tumors (and potentially extrapolated to those with dumbbell tumors) to optimize surgical techniques, benchmark data and share knowledge.

The well known phrase "location, location, location" describes the most important aspects of property values. Similarly, the location of tumors is critical, and the care of our patients is priceless. In unusual locations, many neurosurgeons not only use access surgeons, but rely on them, to bring their expertise and dedicated teams to the multidisciplinary care of our patients. In our practice, we employ specific access surgeons specialized in the surgery at each of these locations.

The authors should be congratulated on their paper and their anatomical contributions to our surgical specialty.

Evelina Llorian,

Robert J. Spinner,

Rochester, Minnesota, USA

The original article has been corrected.

Publisher's note Springer Nature remains neutral with regard to jurisdictional claims in published maps and institutional affiliations. 